\title{
Modelling the Effect of Post-mortem Contact on the Spread of Ebola with Quarantine As an Intervention
}

\author{
Francis T. Oduro, Joseph Baafi \& George Apaaboah \\ Department of Mathematics, Kwame Nkrumah University of Science and Technology Kumasi, Ghana. \\ Correspondence: Francis T. Oduro, Department of Mathematics, Kwame Nkrumah University of Science and Technology \\ Kumasi, Ghana. E-mail: francistoduro@yahoo.co.uk
}

Received: May 25, 2016 Accepted: June 14, 2016 Online Published: August 1, 2016

doi:10.5539/jmr.v8n4p176 URL: http://dx.doi.org/10.5539/jmr.v8n4p176

\begin{abstract}
Ebola virus disease (EVD) is a severe, often fatal disease in humans and other non-human primates caused by infection with any of the four identified Ebola virus species of the family Filoviridae. This paper develops the SEIR and the SEIHDR epidemic models that investigate the effects of the ante-mortem contact and post-mortem contact on the spread of the disease. The reproduction number of the models are determined. The equilibria and conditions for the existence of the equilibria are also determined. The models are solved numerically and the numerical simulations implemented to elucidate various scenarios. The results of the models are then compared to WHO data of confirmed cases for the 2014 Ebola outbreak in Liberia. It is observed that the SEIHDR model agrees better with the data than the SEIR model. Moreover, a new model, the SEIQDR model (a modification of the SEIHDR Model) is formulated which incorporates quarantine as an intervention. Again, this SEIQDR model is compared to the WHO data of confirmed cases for the 2014 Ebola outbreak in Liberia. The results of the SEIQDR model is found to agree better than those of the other models especially in respect of the latter stages of the disease outbreak. Finally, the effect of vaccination on both the SEIHDR and the SEIQDR models is investigated. Different rates of vaccination using numerical simulations in order to predict the effect of vaccination on the infected individuals over time is also discussed. The SEIQDR model with vaccination indicates a lower threshold which should not be less than $25 \%$ as compared to the SEIHDR model for which vaccination should not be less than $65 \%$. It is observed that vaccination as an additional strategy helps to control the disease more effectively.
\end{abstract}

Keywords: Epidemic models, Ebola, post-mortem infectivity, quarantine.

\section{Introduction}

There have been an overwhelming Ebola epidemic in West Africa (Yamin et al., 2015). Ebola virus disease (EVD), which was known as Ebola haemorrhagic fever, is a severe and often fatal disease in human beings and other non-humans such as monkeys and gorillas (WHO, 2015a; CDC, 2015a). The Democratic Republic of Congo happens to be the place where Ebola was first discovered in the year 1976. Since that period, Ebola outbreak have occurred occasionally in several African countries (Legrand et al., 2007; Chowell-Puente, 2005; CDC, 2015a). Ebola spreads among humans through direct contact (through broken skin or mucous membrane) with the blood, secretions, organs or other bodily fluids of infectious individuals (Funk \& Kumar, 2015). Ebola can spread through contact with corpses of Ebola patients, semen or breast milk of a recovered Ebola person (CDC, 2015b; CDC, 2014; Chowell et al., 2015). Persons infected with the virus may experience symptoms as; sudden onset of fever, severe headache, muscle pain, intense weakness, Fatigue, vomiting, sore throat and in some cases both internal and external bleeding (PAHO/WHO, 2014; Gatherer, 2014). Most infected persons may die within 10 days after being infectious. Medicine and knowledge about the disease have advance over the years but EVD outbreaks still pose a treat to humans. Mathematical models have been used by several researchers to study the dynamics of the EVD outbreak but much emphases have not been placed on the post-mortem effect on the spread of the disease and modelling the interventions. It is therefore important that we study and investigate the wide spread of EVD in humans as a result of post-mortem contact and analyse quarantine as an intervention strategy adapted by the health management authorities to control the spread. Thus, the main objective of this work is to study and investigate the effects of post-mortem contact on the spread of Ebola and quarantine as an intervention using mathematical models. We begin by developing the SEIR model adapted from Okeke et al., (2014) to serve as the basis for further investigation. We also study the SEIHDR model adapted from Legrand et al., (2007) which takes into account infection caused by hospitalized individuals and corpses. Again, we formulate the SEIQDR model which is a modification of the previous model to investigate and analyse quarantine as an intervention strategy adapted by the health management authorities to control the spread. We also investigate the effectiveness of vaccination as an intervention strategy. Furthermore, we provide numerical simulations of all the models to illustrate the analytic results using python software. Finally, we compare the 
models to data from Liberia on EVD outbreak.

The paper is organized as follows: In Section 2, we present the SEIR model and its basic reproduction number. We carry out the equilibria and stability analysis of the model and discuss some numerical simulations. In Section 3, we carry out analysis for the SEIHDR model. We also compare the models to an outbreak data in Section 4. Section 5 contains the analysis of the SEIHDR model with vaccination. Section 6 also contains the analysis of the quarantine model (SEIQDR) based on the model in Section 3. In Section 7 we analyse the SEIQDR model with vaccination. Conclusion of this report is given in Section 8.

\section{The SEIR Model}

The SEIR model (adapted from Mamo \& Koya, 2015) is very useful in epidemiology. For example, it has been used by Oduro, (Oduro, 2012) to study the transmission dynamics of Malaria in Ghana and also in the study of Ebola transmission dynamics (Oduro, 2016). In this model, the total population $(\mathrm{N})$ is categorised into four compartments namely, the susceptible (S), the exposed (E), the infectious (I), and the removed (R) (Keeling \& Rohani, 2008).

The susceptibles are uninfected individuals who may become exposed after contact with an infectious individual. The exposed comprises of individuals, who have been infected but not yet infectious. The infectious individuals are those with the disease and can transmit the infection to other susceptible individuals. The removed comprises of individuals removed from the chain of transmission either by recovery or dead and buried. At any given time (t), the number of individuals in the susceptible, exposed, infectious and removed compartment is denoted by $\mathrm{S}(\mathrm{t}), \mathrm{E}(\mathrm{t}), \mathrm{I}(\mathrm{t})$ and $\mathrm{R}(\mathrm{t})$ respectively (Britton, 2012; Rivers et al., 2014).

\subsection{Some Assumptions Underlying the Model}

The model makes the following assumptions:

- All individuals in the population are initially considered susceptible ie. $\mathrm{N}(0)=\mathrm{S}(0)$.

- The recruitment rate is equal to the natural death rate.

- Individuals become infectious, by first becoming exposed.

- Individuals leave the infectious class either by recovery, dead and buried or due to natural death (and move to class $\mathrm{R})$.

- Age, sex, status and race do not affect the probability of being infected with the Ebola virus.

- All cases of infection are assumed to be related to human-to-human transmission.

\subsection{The Compartmental Model}

In this section, we consider the SEIR compartmental model. The effective contact between a susceptible and an infectious individual is denoted by $\beta$. The parameter $b$ is the recruitment or natural death rate that may occur in the population under consideration. The asymptomatic non-infectious exposed individual eventually show the symptoms of the ebola disease and pass onto the infectious class. This is denoted by $\gamma E$, where $\gamma$ is the per-capita infectious rate. Thus the average duration for an individual to be infectious is $1 / \gamma . \delta$ is the death or recovery rate of an infected individual. As before, death and recovery are considered to be in the same compartment, since there has not yet been any reported case where an individual who survived Ebola contracts the virus again (Astacio et al., 1996).

The above model together with the assumptions can be expressed in mathematical terms by the following system of ordinary differential equations:

$$
\begin{aligned}
S^{\prime} & =b N-\beta S \frac{I}{N}-b S, \\
E^{\prime} & =\beta S \frac{I}{N}-(\gamma+b) E, \\
I^{\prime} & =\gamma E-(\delta+b) I, \\
R^{\prime} & =\delta I-b R,
\end{aligned}
$$

with the non-negativity requirements of the initial conditions:

$$
S(0) \geq 0, \quad E(0) \geq 0, \quad I(0) \geq 0, \quad R(0) \geq 0 .
$$




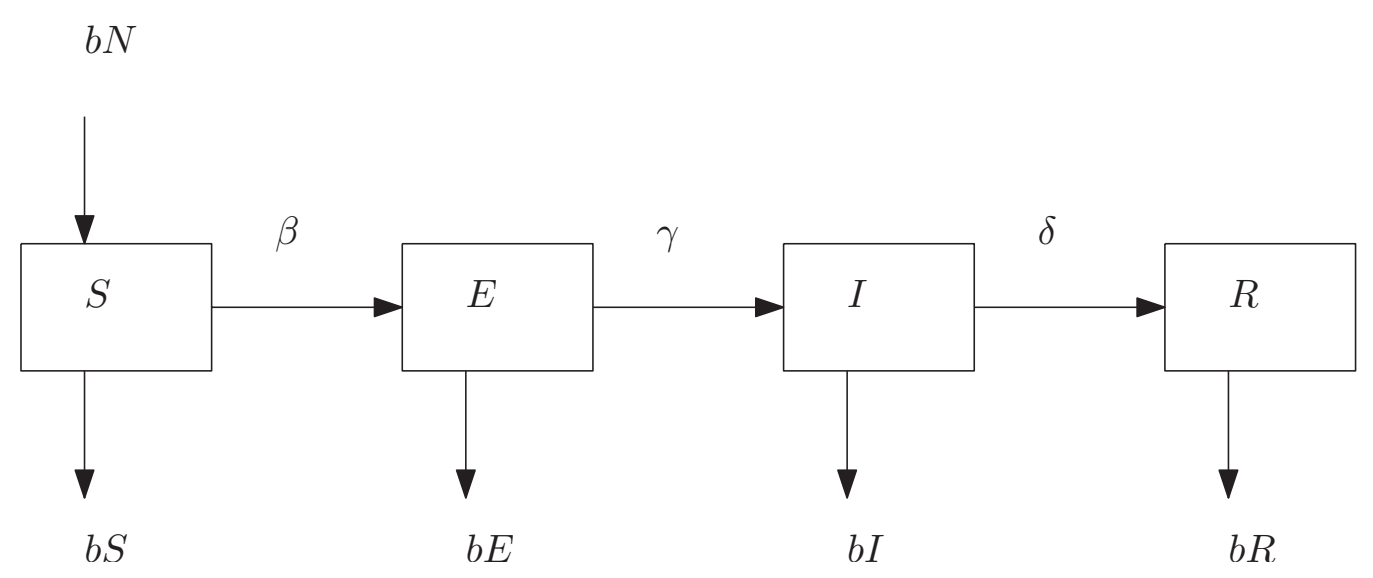

Figure 1. SEIR compartmental model indicating the movement of individuals from one class to another.

$\mathrm{N}$ is the total population under consideration. Thus the total population density is given by $S(t)+E(t)+I(t)+R(t)=N(t)$. Adding the four equations, we see that

$$
N^{\prime}=S^{\prime}+E^{\prime}+I^{\prime}+R^{\prime}=0,
$$

thus $N(t)=N(0)$ and so the population size is constant. Given non-negative initial data (2), it is easy to check that for all $t>0$, the solution always lies in the set $\mathcal{I}$ :

$$
\mathcal{I}=\{(S, E, I, R) \mid S(t) \geq 0, E(t) \geq 0, \quad I(t) \geq 0, R(t) \geq 0, \quad S(t)+E(t)+I(t)+R(t)=N\} .
$$

\subsection{The Basic Reproduction Number}

This section determines the basic reproduction number $R_{0}$. This is an important quantity which determines whether a disease persists or dies out and also how fast it spreads. It is defined as the average number of secondary cases arising from a single infective introduced into an entirely susceptible population of size $N$ (Astacio et al., 1996; Keeling \& Rohani, 2008). We use the method of next generation matrix to determine the expression for $R_{0}$ (Driessche \& Watmough, 2002; Khanh, 2015; Khan et. al., 2015). From the equilibrium analysis carried out in 2.4 of this section, the system (1) has a disease-free equilibrium (DFE) given by $(N, 0,0,0)$. The infected compartments are $E$ and $I$. Let $x=(E, I, S, R)^{T}$. Then the system (1) can be written as

$$
\frac{d x}{d t}=\mathcal{F}(x)-\mathcal{V}(x)
$$

where

$$
\mathcal{F}(x)=\left(\begin{array}{c}
\frac{\beta S I}{N} \\
0 \\
0 \\
0
\end{array}\right) \quad \text { and } \quad \mathcal{V}(x)=\left(\begin{array}{c}
(\gamma+b) E \\
-\gamma E+(\delta+b) I \\
-b N+\frac{\beta S I}{N}-b S \\
-\delta I+b R
\end{array}\right)
$$

This gives the following:

$$
\begin{aligned}
& F=\text { Jacobian of } \mathcal{F} \text { at DFE }=\left(\begin{array}{ll}
0 & \beta \\
0 & 0
\end{array}\right), \\
& V=\text { Jacobian of } \mathcal{V} \text { at DFE }=\left(\begin{array}{cc}
(\gamma+b) & 0 \\
-\gamma & (\delta+b)
\end{array}\right) .
\end{aligned}
$$

Thus,

$$
V^{-1}=\left(\begin{array}{cc}
\frac{\delta+b}{(\delta+b)(\gamma+b)} & 0 \\
\gamma & \gamma+b
\end{array}\right)
$$


The next generation matrix for the system (1) is

$$
F V^{-1}=\left(\begin{array}{cc}
\frac{\beta \gamma}{(\delta+b)(\gamma+b)} & \frac{\beta(\gamma+b)}{(\delta+b)(\gamma+b)} \\
0 & 0
\end{array}\right)
$$

We have the spectral radius of the matrix $F V^{-1}$ to be the reproduction number of the system (1) according to (Driessche $\&$ Watmough, 2001). Thus, the basic reproduction number is given by:

$$
R_{0}=\frac{\beta \gamma}{(\delta+b)(\gamma+b)}
$$

$R_{0}$ is the critical parameter in determining the stability of equilibria for the above system.

\subsection{Equilibria}

The model (1) has two equilibria, the disease free and the endemic equilibria. It can be shown (Keeling \& Rohani, 2008) that the disease free equilibrium given by

$$
\left(S^{*}, E^{*}, I^{*}, R^{*}\right)=(N, 0,0,0),
$$

exists and the endemic equilibrium is given by

$$
\left(S^{*}, E^{*}, I^{*}\right)=\left(\frac{N}{R_{0}}, \frac{b N(\delta+b)}{\beta \gamma}\left(R_{0}-1\right), \frac{b N}{\beta}\left(R_{0}-1\right)\right) .
$$

\subsection{Stability Analysis}

In this section, we discuss the stability of the disease free and endemic equilibria. In system (1), the fourth equation is independent of the rest, so we can omit it and reduce the system to a set of three equations:

$$
\begin{aligned}
S^{\prime} & =b N-\beta S \frac{I}{N}-b S, \\
E^{\prime} & =\beta S \frac{I}{N}-(\gamma+b) E, \\
I^{\prime} & =\gamma E-(\delta+b) I .
\end{aligned}
$$

We study the stability by stating and proving these theorems:

Theorem 2.1. If $R_{0}<1$, then the disease free equilibrium (DFE) is stable.

Proof. The Jacobian matrix of the system (4) is given by:

$$
J=\left(\begin{array}{ccc}
-\frac{\beta I^{*}}{N}-b & 0 & -\frac{\beta S^{*}}{N} \\
\frac{\beta I^{*}}{N} & -(\gamma+b) & \frac{\beta S^{*}}{N} \\
0 & \gamma & -(\delta+b)
\end{array}\right) .
$$

Now we evaluate this at the disease-free equilibrium. The Jacobian matrix evaluated at the disease-free equilibrium gives:

$$
J(D F E)=\left(\begin{array}{ccc}
-b & 0 & -\beta \\
0 & -(\gamma+b) & \beta \\
0 & \gamma & -(\delta+b)
\end{array}\right) .
$$

We compute the eigenvalues of this matrix as follows:

$$
\begin{gathered}
\left|\begin{array}{ccc}
-b-\lambda & 0 & -\beta \\
0 & -(\gamma+b)-\lambda & \beta \\
0 & \gamma & -(\delta+b)-\lambda
\end{array}\right|=0, \\
\Rightarrow(-b-\lambda)[(\gamma+b+\lambda)(\delta+b+\lambda)-\beta \gamma]=0,
\end{gathered}
$$


thus

$$
\lambda_{1}=-b
$$

and

$$
\begin{aligned}
(\gamma+b+\lambda)(\delta+b+\lambda)-\beta \gamma & =0, \\
\Rightarrow \lambda^{2}+(2 b+\gamma+\delta) \lambda+\left(\gamma \delta+b \gamma+b \delta+b^{2}-\beta \gamma\right) & =0 .
\end{aligned}
$$

We solve the quadratic equation as follows:

$$
\begin{aligned}
\lambda & =\frac{-(2 b+\gamma+\delta) \pm \sqrt{(2 b+\gamma+\delta)^{2}-4\left(\gamma \delta+b \gamma+b \delta+b^{2}-\beta \gamma\right)}}{2} \\
& =\frac{-(2 b+\gamma+\delta) \pm \sqrt{(2 b+\gamma+\delta)^{2}-4[(b+\delta)(b+\gamma)-\beta \gamma]}}{2} \\
& =\frac{-(2 b+\gamma+\delta) \pm \sqrt{(2 b+\gamma+\delta)^{2}-4(b+\delta)(b+\gamma)\left(1-\frac{\beta \gamma}{(b+\delta)(b+\gamma)}\right)}}{2} \\
& =\frac{-(2 b+\gamma+\delta) \pm \sqrt{(2 b+\gamma+\delta)^{2}-4(b+\delta)(b+\gamma)\left(1-R_{0}\right)}}{2}
\end{aligned}
$$

Thus we have that:

$$
\lambda_{2}=\frac{-(2 b+\gamma+\delta)+\sqrt{(2 b+\gamma+\delta)^{2}-4(b+\delta)(b+\gamma)\left(1-R_{0}\right)}}{2}
$$

and

$$
\lambda_{3}=\frac{-(2 b+\gamma+\delta)-\sqrt{(2 b+\gamma+\delta)^{2}-4(b+\delta)(b+\gamma)\left(1-R_{0}\right)}}{2},
$$

are the eigenvalues at the disease-free equilibrium. If $R_{0}<1$ then

$$
\lambda_{2}<-\frac{2 b+\gamma+\delta}{2}+\frac{\sqrt{(2 b+\gamma+\delta)^{2}}}{2}=0
$$

and

$$
\lambda_{3}<-\frac{2 b+\gamma+\delta}{2}-\frac{\sqrt{(2 b+\gamma+\delta)^{2}}}{2}=-(2 b+\gamma+\delta) .
$$

Since all the eigenvalues have negative real parts for $R_{0}<1$, the disease-free equilibrium is stable.

Theorem 2.2. If $R_{0}>1$, then the endemic equilibrium (EE) is stable.

Proof. The Jacobian matrix at the endemic equilibrium (EE) is given by:

$$
J(E E)=\left(\begin{array}{ccc}
-b R_{0} & 0 & -\frac{\beta}{R_{0}} \\
b\left(R_{0}-1\right) & -(\gamma+b) & \frac{\beta}{R_{0}} \\
0 & \gamma & -(\delta+b)
\end{array}\right) .
$$

Now we compute the eigenvalues of the endemic equilibrium point as follows:

$$
\begin{aligned}
\left|\begin{array}{ccc}
-b R_{0}-\lambda & 0 & -\frac{\beta}{R_{0}} \\
b\left(R_{0}-1\right) & -(\gamma+b)-\lambda & \frac{\beta}{R_{0}} \\
0 & \gamma & -(\delta+b)-\lambda
\end{array}\right|=0, & \\
\Rightarrow-\left(b R_{0}+\lambda\right)\left[(\gamma+b+\lambda)(\delta+b+\lambda)-\frac{\beta \gamma}{R_{0}}\right]-\frac{\beta}{R_{0}}\left(b \gamma\left(R_{0}-1\right)\right) & =0, \\
-\left(b R_{0}+\lambda\right)[(\gamma+b+\lambda)(\delta+b+\lambda)-(b+\delta)(b+\gamma)]-b\left(R_{0}-1\right)(b+\delta)(b+\gamma) & =0, \\
-\left(b R_{0}+\lambda\right)\left[\lambda^{2}+(\gamma+2 b+\delta) \lambda\right]-b\left(R_{0}-1\right)(b+\delta)(b+\gamma) & =0 .
\end{aligned}
$$


Expanding and simplifying the algebra, we have

$$
\lambda^{3}+\left(b R_{0}+2 b+\gamma+\delta\right) \lambda^{2}+b R_{0}(2 b+\gamma+\delta) \lambda+b\left(R_{0}-1\right)(b+\delta)(b+\gamma)=0 .
$$

This is in the form

$$
\lambda^{3}+a_{1} \lambda^{2}+a_{2} \lambda+a_{3}=0
$$

where

$$
\begin{aligned}
& a_{1}=b R_{0}+2 b+\gamma+\delta, \\
& a_{2}=b R_{0}(2 b+\gamma+\delta), \\
& a_{3}=b\left(R_{0}-1\right)(b+\delta)(b+\gamma) .
\end{aligned}
$$

We use the RouthCHurwitz stability criterion to determine the stability of the endemic equilibrium. It can be seen that if $R_{0}>1$, we have

$$
a_{1}>0, a_{2}>0, a_{3}>0 .
$$

Also

$$
a_{1} a_{2}-a_{3}=\left(b R_{0}+2 b+\gamma+\delta\right)\left(b R_{0}(2 b+\gamma+\delta)\right)-b\left(R_{0}-1\right)(b+\delta)(b+\gamma)>0 .
$$

Thus all the eigenvalues have negative real parts by the RouthCHurwitz criteria. Hence the endemic equilibrium of the system (4) is stable.

\subsection{Numerical Simulations}

In this section we solve the system of differential equations (1) numerically. A crucial parameter which governs the behaviour of the system is the reproduction number $R_{0}$ which is given in (3). In all the graphs, we have time (measured in years) on the horizontal axis and the proportion of the population who are either susceptible, exposed, infectious or removed (ie. $s=\frac{S}{N}, e=\frac{E}{N}, i=\frac{I}{N}, r=\frac{R}{N}$ so that the total population is $N=1$ ) is on the vertical axis. All simulations are produced using python (odeint).

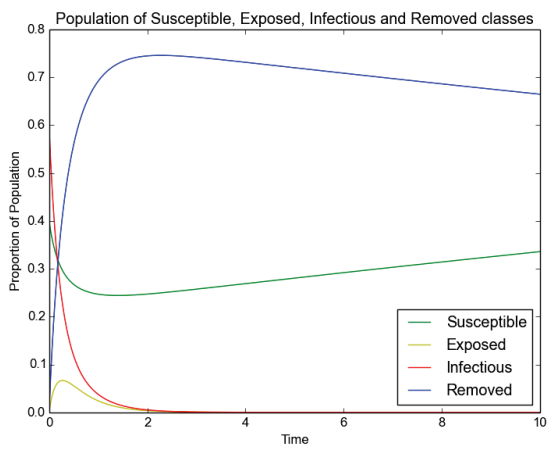

(a)

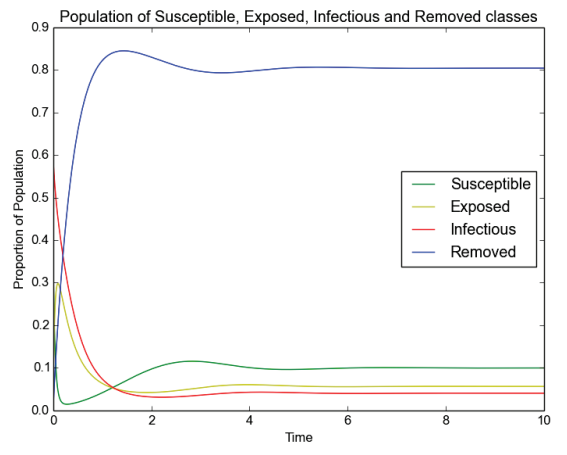

(b)

Figure 2. Numerical solutions of the SEIR model (1) with $b=0.016, \quad \beta=3, \quad \gamma=3, \quad \delta=4, \quad N=1, \quad s(0)=$ $0.4, e(0)=0, i(0)=0.6, r(0)=0$ and $R_{0}=0.74<1$. By Theorem 2.1 the disease-free equilibrium is stable (a). Also, $b=0.2, \beta=45, \gamma=3, \delta=4, N=1, s(0)=0.8, e(0)=0, i(0)=0.2, r(0)=0$, and $R_{0}=10.04>1$. By Theorem 2.2 , the endemic equilibrium is stable (b).

\section{The SEIHDR Model}

In the previous section 2, we studied the SEIR model. The major assumption was that when an infective dies (either natural or disease-related), he or she is removed from the chain of transmission. However, disease-related death does not end transmission but causes infection upon contact with a susceptible individual. To better understand the dynamics of the disease it is important to consider the dead bodies as a means of transfer of disease. In this regard a model called the SEIHDR model was developed by Legrand et al. (2006). The variables H and D represent infectives who become hospitalized and dead respectively. 


\subsection{Model Assumptions}

The model accounts for two aspects of disease transfer.

(i) Infection at the hospitals as a result of health workers interaction with hospitalised individuals.

(ii) Infection caused by dead bodies of Ebola patients during funerals and burial services.

Table 1 is a description of the parameters that we use in the SEIHDR model:

Table 1. Model Parameters and their description (Rivers et. al., 2014).

\begin{tabular}{|l|l|}
\hline Parameter & Description \\
\hline$\mu$ & Recruitment rate of susceptible individuals \\
$\beta_{I}$ & Contact rate in the Community \\
$\beta_{H}$ & Contact rate in the Hospital \\
$\beta_{D}$ & Contact rate during Funeral \\
$1 / \gamma$ & Incubation period \\
$1 / \alpha_{H}$ & Time until Hospitalization \\
$1 / \alpha_{D H}$ & Time from Hospitalization to Death \\
$1 / \alpha_{F}$ & Duration of traditional Funeral \\
$1 / \alpha_{I}$ & Duration of Infection \\
$1 / \alpha_{D}$ & Time from Infection to Death \\
$1 / \alpha_{I H}$ & Time from Hospitalization to Removed \\
$\theta_{1}$ & Fraction of Infected Hospitalized \\
$\delta_{1}$ & Case fatality rate, Unhospitalized \\
$\delta_{2}$ & Case fatality rate, Hospitalized \\
\hline
\end{tabular}

Figure 3 represents the compartmental SEIHDR model.

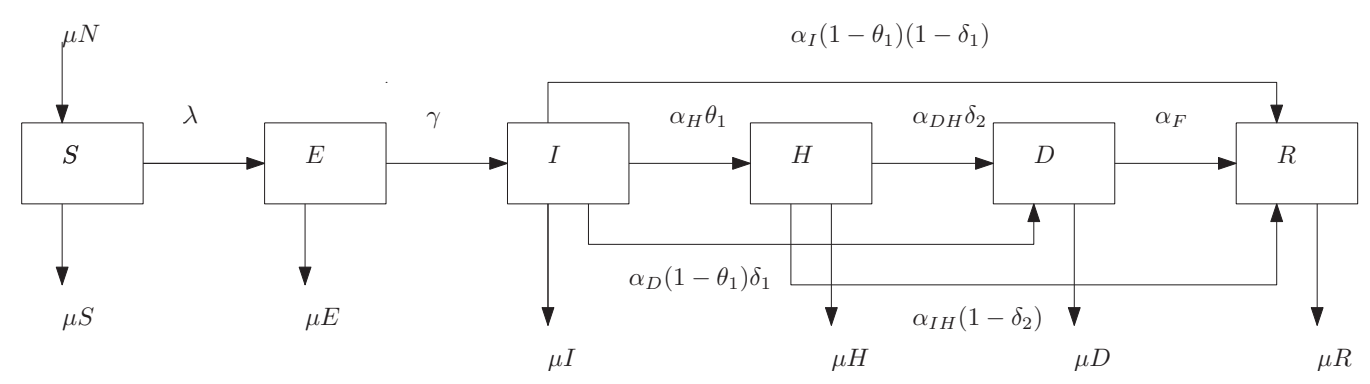

Figure 3. The SEIHDR compartmental model which represents the dynamics of the Ebola virus disease in a flow diagram.

This model can be represented by the following system of differential equations.

$$
\begin{aligned}
S^{\prime} & =\mu N-\frac{\beta_{I} S I+\beta_{H} S H+\beta_{D} S D}{N}-\mu S, \\
E^{\prime} & =\frac{\beta_{I} S I+\beta_{H} S H+\beta_{D} S D}{N}-(\gamma+\mu) E, \\
I^{\prime} & =\gamma E-\left(\alpha_{H} \theta_{1}+\alpha_{D}\left(1-\theta_{1}\right) \delta_{1}+\alpha_{I}\left(1-\theta_{1}\right)\left(1-\delta_{1}\right)+\mu\right) I, \\
H^{\prime} & =\alpha_{H} \theta_{1} I-\left(\alpha_{D H} \delta_{2}+\alpha_{I H}\left(1-\delta_{2}\right)+\mu\right) H, \\
D^{\prime} & =\alpha_{D}\left(1-\theta_{1}\right) \delta_{1} I+\alpha_{D H} \delta_{2} H-\left(\alpha_{F}+\mu\right) D, \\
R^{\prime} & =\alpha_{I}\left(1-\theta_{1}\right)\left(1-\delta_{1}\right) I+\alpha_{I H}\left(1-\delta_{2}\right) H+\alpha_{F} D-\mu R,
\end{aligned}
$$

with the non-negativity requirements of the initial conditions:

$$
S(0) \geq 0, \quad E(0) \geq 0, \quad I(0) \geq 0, \quad H(0) \geq 0, \quad D(0) \geq 0, \quad R(0) \geq 0 .
$$

The system (5) has both disease-free and endemic equilibria. Analysis of these equilibria are similar to that of the model in section 2 . 


\subsection{Numerical Simulations}

In this section, we study system (5) numerically. We show some numerical simulations. We have time (measured in years) on the horizontal axis and the proportion of the population who are either susceptible, exposed, infectious, hospitalized, dead or removed (ie. $s=\frac{S}{N}, e=\frac{E}{N}, i=\frac{I}{N}, h=\frac{H}{N}, d=\frac{D}{N}, r=\frac{R}{N}$ so that the total population is $N=1$ ) is on the vertical axis.

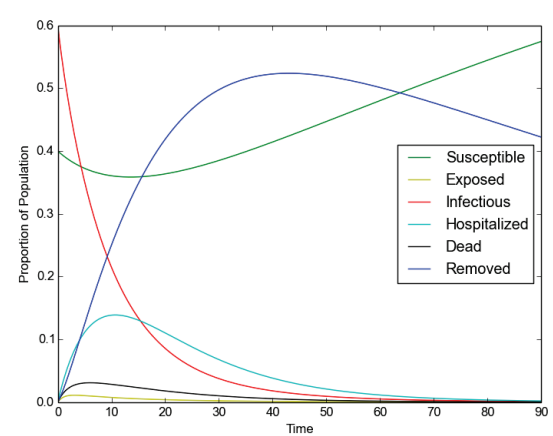

(a)

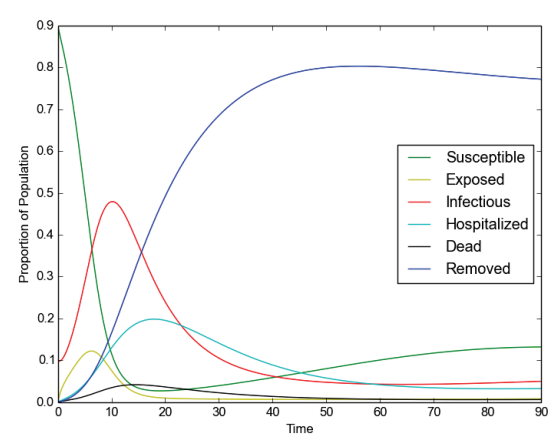

(b)

Figure 4. Numerical simulations of the SEIHDR model (1) with $\mu=0.007, \beta_{I}=0.050, \beta_{H}=0.034, \beta_{D}=0.012, \gamma=$ $0.83, \alpha_{H}=0.309, \alpha_{D}=0.075, \alpha_{I}=0.067, \alpha_{D H}=0.099, \alpha_{I H}=0.063, \theta_{1}=0.197, \delta_{1}=0.50, \delta_{2}=0.50, \quad N=$ $1, \quad s(0)=0.4, \quad e(0)=0, \quad i(0)=0.6, \quad h(0)=0, d(0)=0, \quad r(0)=0$ and $R_{0}^{*}=0.659<1$ (a). Also, $\mu=0.008, \beta_{I}=$ $0.650, \beta_{H}=0.540, \beta_{D}=0.330, \gamma=0.83, \alpha_{H}=0.309, \alpha_{D}=0.075, \alpha_{I}=0.067, \alpha_{D H}=0.099, \alpha_{I H}=0.063, \theta_{1}=$ $0.197, \quad \delta_{1}=0.50, \quad \delta_{2}=0.50, \quad N=1, \quad s(0)=0.9, \quad e(0)=0, \quad i(0)=0.1, \quad h(0)=0, \quad d(0)=0, \quad r(0)=0$, and $R_{0}^{*}=10.02>1(\mathrm{~b})$.

\section{Comparison of the SEIR and the SEIHDR Models to WHO Data}

We compare both the SEIR model and the SEIHDR model to the WHO data of confirmed cases for the 2014 Ebola outbreak in Liberia (WHO, 2015b). In Figure 5 we compare the infected I(t) obtained from the SEIR model to the WHO data. Also in Figure 6 we compare the infected I(t) from the SEIHDR model to the WHO data. The choice of the initial conditions are as a result of the data shown in Figure 2 of the Ebola response roadmap situation report by the WHO (WHO, 2015c; Rachah \& Torres, 2015) with most parameter values taken from Rivers et. al. (2014).

It is seen from Figure 5 and 6 that the SEIHDR model agrees better with the data especially during the early stages of the outbreak than that of the SEIR model. This makes sense since the post-mortem contact was a major means of disease spread during the early stages due to misunderstanding and misdiagnosis of the Ebola virus. This confirms that postmortem contact effect is important in the dynamics of the disease during the early stages. It is worth noticing that after interventions were introduced the model no longer agrees with the data. In the next section, we consider quarantine as an intervention.

\section{The SEIHDR Model with Vaccination}

Another strategy to control the spread of infectious diseases is through vaccination. Now our objective is to study the effect of vaccination in an Ebola disease outbreak. The situation where the French nurse was cured of Ebola with the help of an experimental vaccine is a proof of the fact that vaccinating susceptible individuals is a means to control further spread (Rachah \& Torres, 2015).

\subsection{The Compartmental Model with Vaccination}

In this section, we modify the SEIHDR model in section 3 by adding a vaccination term $v$. Figure 7 shows the relationship between the variables of the SEIHDR model with vaccination. 


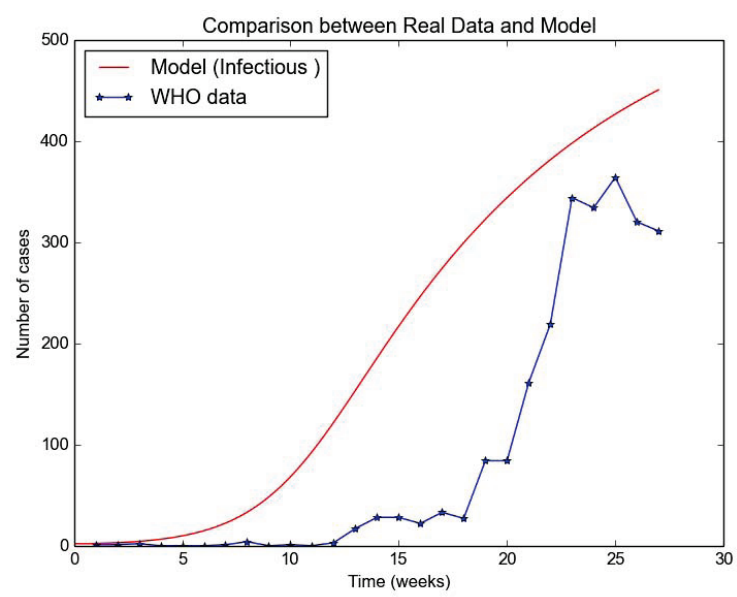

Figure 5. Graph of infected I(t) obtained from (1) with $b=0.0001, \beta=0.0047, \gamma=0.083, \delta=0.00163, S(0)=600$, $E(0)=0, I(0)=2$, and $R(0)=0$ versus the real data of confirmed cases for the 2014 Ebola outbreak in Liberia from March 30 to October 4, 2014.

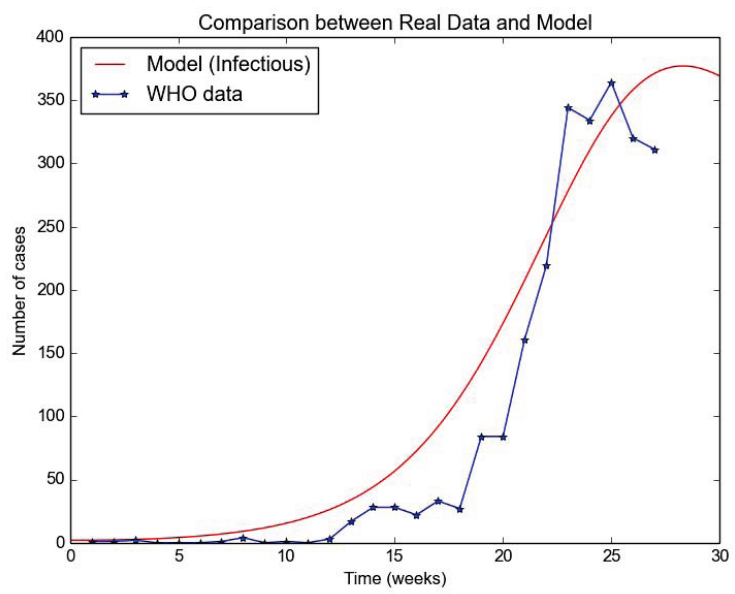

Figure 6. Graph of infected I(t) obtained from (5) with $\mu=0.0001, \beta_{I}=0.0002, \beta_{H}=0.0035, \beta_{D}=0.0010, \gamma=0.83$, $\alpha_{H}=0.28, \alpha_{D}=0.095, \alpha_{I}=0.002, \alpha_{D H}=0.099, \alpha_{I H}=0.063, \theta_{1}=0.185, \delta_{1}=0.0003, \delta_{2}=0.500, S(0)=600$, $E(0)=0, I(0)=2, H(0)=0, D(0)=0$, and $R(0)=0$ versus the real data of confirmed cases for the 2014 Ebola outbreak in Liberia from March 30 to October 4, 2014.

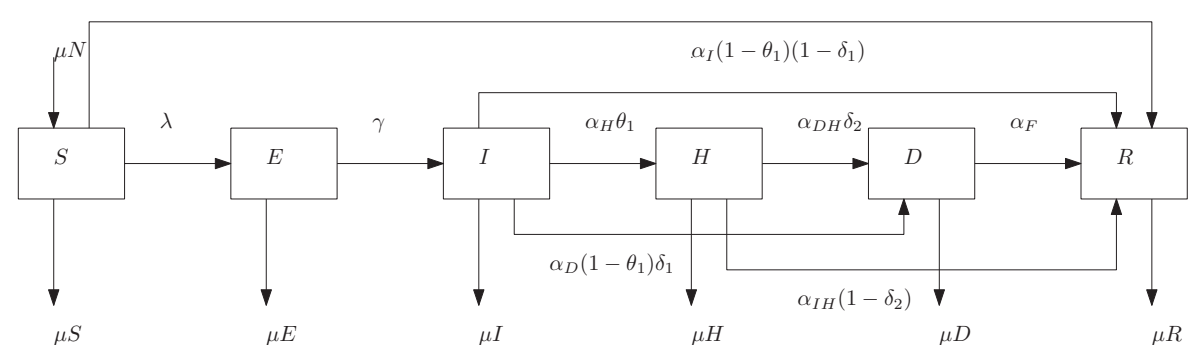

Figure 7. The SEIHDR compartmental model with vaccination.

The system of equations that describe the SEIHDR model with vaccination are: 


$$
\begin{aligned}
S^{\prime} & =\mu N-\frac{\beta_{I} S I+\beta_{H} S H+\beta_{D} S D}{N}-\mu S-v S, \\
E^{\prime} & =\frac{\beta_{I} S I+\beta_{H} S H+\beta_{D} S D}{N}-(\gamma+\mu) E, \\
I^{\prime} & =\gamma E-\left(\alpha_{H} \theta_{1}+\alpha_{D}\left(1-\theta_{1}\right) \delta_{1}+\alpha_{I}\left(1-\theta_{1}\right)\left(1-\delta_{1}\right)+\mu\right) I, \\
H^{\prime} & =\alpha_{H} \theta_{1} I-\left(\alpha_{D H} \delta_{2}+\alpha_{I H}\left(1-\delta_{2}\right)+\mu\right) H, \\
D^{\prime} & =\alpha_{D}\left(1-\theta_{1}\right) \delta_{1} I+\alpha_{D H} \delta_{2} H-\left(\alpha_{F}+\mu\right) D, \\
R^{\prime} & =\alpha_{I}\left(1-\theta_{1}\right)\left(1-\delta_{1}\right) I+\alpha_{I H}\left(1-\delta_{2}\right) H+\alpha_{F} D-\mu R+v S,
\end{aligned}
$$

where $v$ is the percentage of individuals vaccinated.

\subsection{Numerical Simulations in Case of Vaccination}

We simulate the SEIHDR Model with vaccination (7) in order to visualise and predict the evolution of every class of individuals in case of vaccination (Rachah \& Torres, 2015). This study focusses on the test of different rates of vaccination and how they affect the curve of every class. To simplify the system, we will consider the proportion of susceptible, exposed, infectious, hospitalized, dead or removed (ie. $s=\frac{S}{N}, e=\frac{E}{N}, i=\frac{I}{N}, h=\frac{H}{N}, d=\frac{D}{N}, r=\frac{R}{N}$ so that the total population is $N=1$ )

The results are shown in Figures 9 and 10. These figures show different rates of vaccination and their effect on the peak of the curve of the infected individuals with time. The curve of the infected class shows that when the percentage of vaccinated individuals increases the peak of the curve of infected class goes down indicating that less individuals are infected and the period of infection is shortened while the number of removed individuals go up.

Table 2 shows the percentage of infected individuals i corresponding to different rates of vaccination $\mathrm{v}$ and the respective number of weeks for the peak of the $\mathrm{i}(\mathrm{t})$ curve to be reached.

Table 2. Maximum percentage (to the nearest whole number) infected i corresponding to different rates of vaccination $v$ and the respective number of weeks for the peak of the $i(t)$ curve to be reached.

\begin{tabular}{|c|c|c|}
\hline Rate of vaccination & Maximum percentage infected & Weeks to the peak of $\mathrm{i}(\mathrm{t})$ \\
\hline 0 & $48 \%$ & 10.14 \\
0.10 & $28 \%$ & 8.88 \\
0.20 & $20 \%$ & 7.10 \\
0.40 & $14 \%$ & 4.77 \\
0.50 & $13 \%$ & 4.24 \\
0.60 & $12 \%$ & 3.52 \\
0.70 & $11 \%$ & 3.34 \\
0.80 & $11 \%$ & 2.99 \\
\hline
\end{tabular}

It can be seen from Table 2 that as the rate of vaccination increases the maximum percentage of individuals infected also increases. Increasing $v$ between 0.6 and 1.0, the percentage infected increases by a small margin. Figure 8 shows the relation between the rate of vaccination $v$ and the maximum individuals infected $i$ by the disease.

From Figure 8, the threshold proportion of population to be vaccinated to control the disease is estimated to be not less than $v=0.75$. 


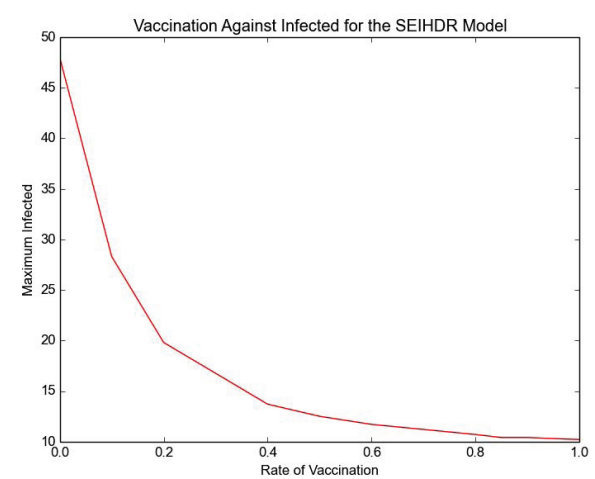

Figure 8. A graph showing the relation between the rate of vaccination and the maximum percentage of individuals infected for the SEIHDR model.

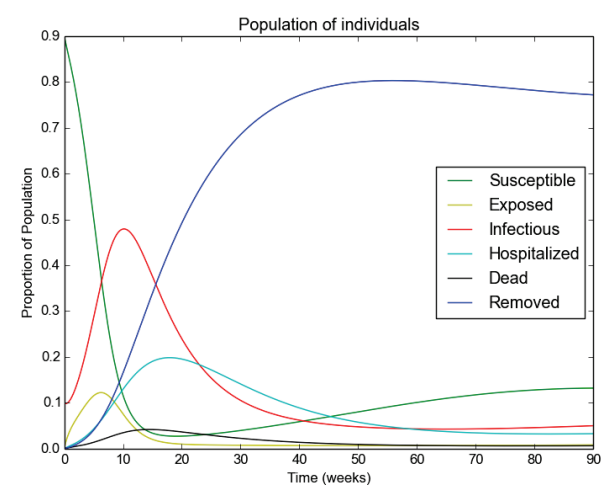

(a)

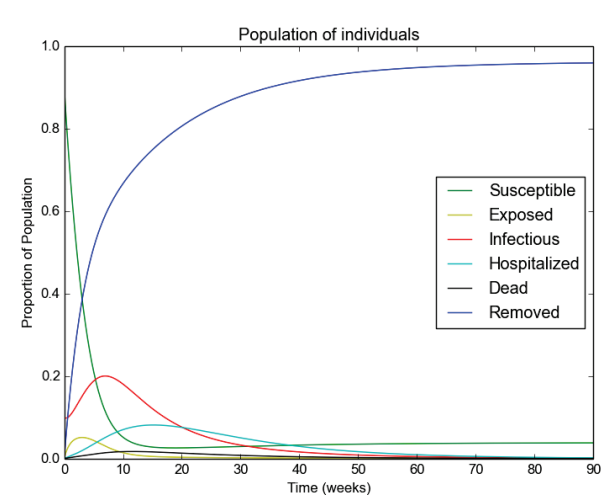

(c)

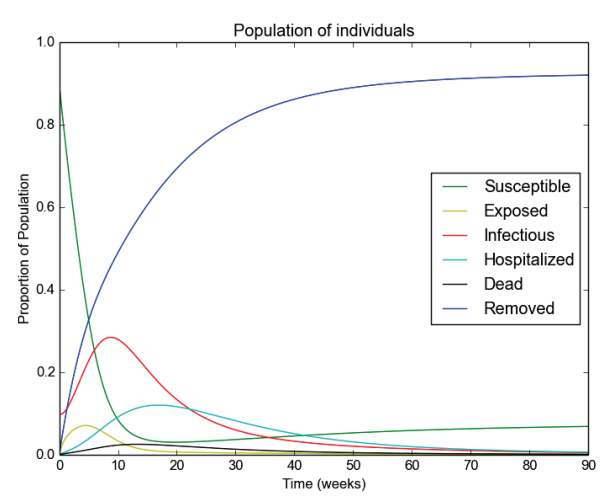

(b)

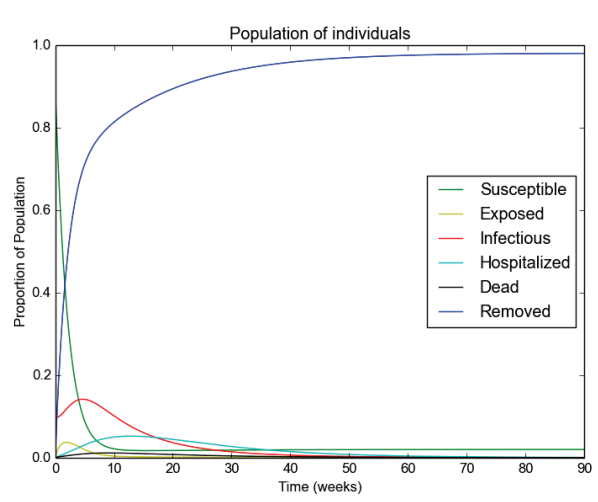

(d)

Figure 9. Numerical simulations of the SEIHDR model with parameter values same as that of Figure 12 and different rates of vaccination: $v=0$ (a), $v=0.10$ (b), $v=0.20$ (c), $v=0.40$ (d).

\section{The SEIQDR Model (A Modified SEIHDR Model)}

\subsection{Formulation of the SEIQDR Model}

The major strategy adopted to control the spread of the Ebola virus disease is to isolate exposed and infectious (and dead) individuals to prevent further contact and possible subsequent transmission. In this section, we modify the SEIHDR model through the equations (5) in Section 3 by introducing the compartment $\mathrm{Q}$ to denote the exposed and infectious population being quarantined which for our purposes includes hospitalized individuals. We also introduces new parameters as shown 


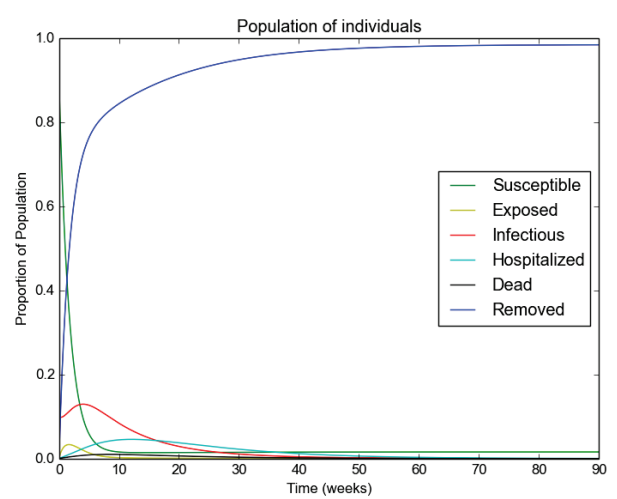

(a)

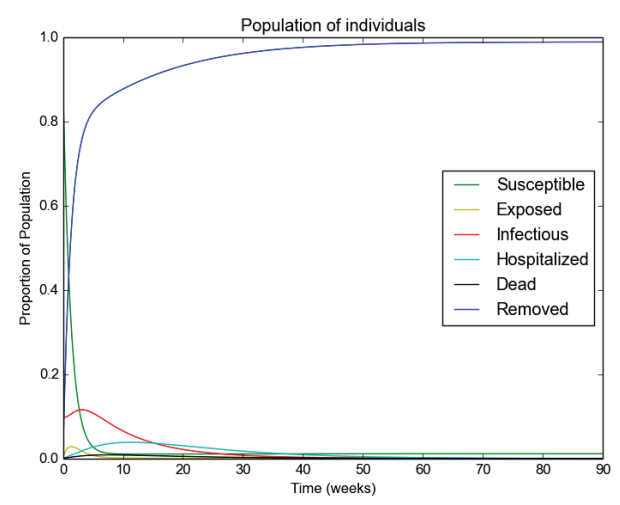

(c)

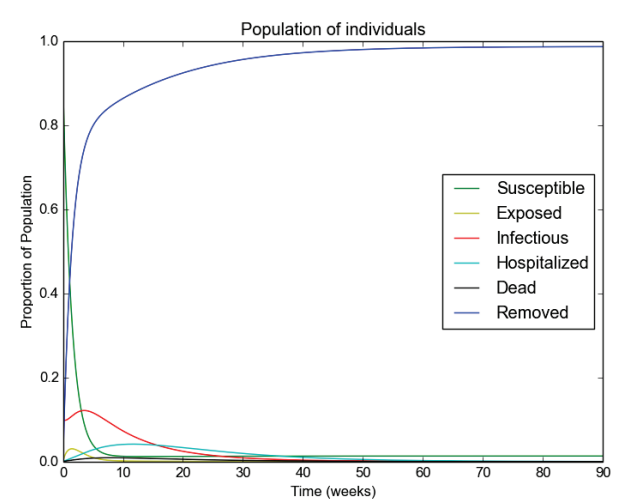

(b)

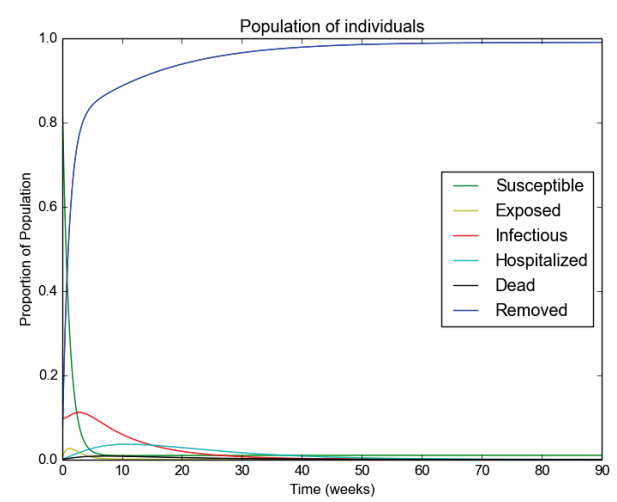

(d)

Figure 10. Numerical simulations of the SEIHDR model with parameter values same as that of Figure 12 and different rates of vaccination: $v=0.50(\mathrm{a}), v=0.60(\mathrm{~b}), v=0.70(\mathrm{c}), v=0.80(\mathrm{~d})$.

in Table 3. We call this modification of the SEIHDR model the SEIQDR model.

\subsection{New Model Parameters}

Table 3 is a description of the new parameters of the SEIQDR model:

\subsection{The Compartmental Model}

In this section, we consider the compartmental SEIQDR model given in Figure 11. From the previous model (5),

$$
\lambda=\beta_{I}+\beta_{H}+\beta_{D}
$$

but for this model,

$$
\lambda=\beta_{I}+\beta_{D} .
$$

This is because quarantined individuals do not interact with susceptible ones. Also, $\beta_{D}$ is the contact rate between nonquarantined dead bodies of Ebola patients and susceptible individuals during funerals. Quarantine individuals are given proper burial and so do not contribute to further spread. Thus $\beta_{D}$ has a lower value as compared to that of model (5). It can be seen that the rate of transmission of infection in model (10) is reduced as compared to the previous models as a result of the method of isolation of infected individuals. We analyse the effectiveness of this mode of infection control in the subsequent sections.

The model can be represented by the following system of ordinary differential equations: 
Table 3. Model Parameters and their description.

\begin{tabular}{|l|l|}
\hline Parameter & Description \\
\hline $1 / \gamma_{Q}$ & Time from Exposed to Quarantine \\
$1 / \gamma_{I}$ & Time from Exposed to Infected \\
$1 / \alpha_{Q}$ & Time from Infected to Quarantine \\
$1 / \alpha_{D Q}$ & Time from Quarantine to Death \\
$1 / \alpha_{I Q}$ & Time from Quarantine to Removed \\
$\tau$ & Fraction of Exposed Infected \\
$\theta_{1}$ & Fraction of Infected Quarantined \\
$\delta_{1}$ & Case fatality rate, Un-quarantined \\
$\delta_{2}$ & Case fatality rate, Quarantined \\
\hline
\end{tabular}

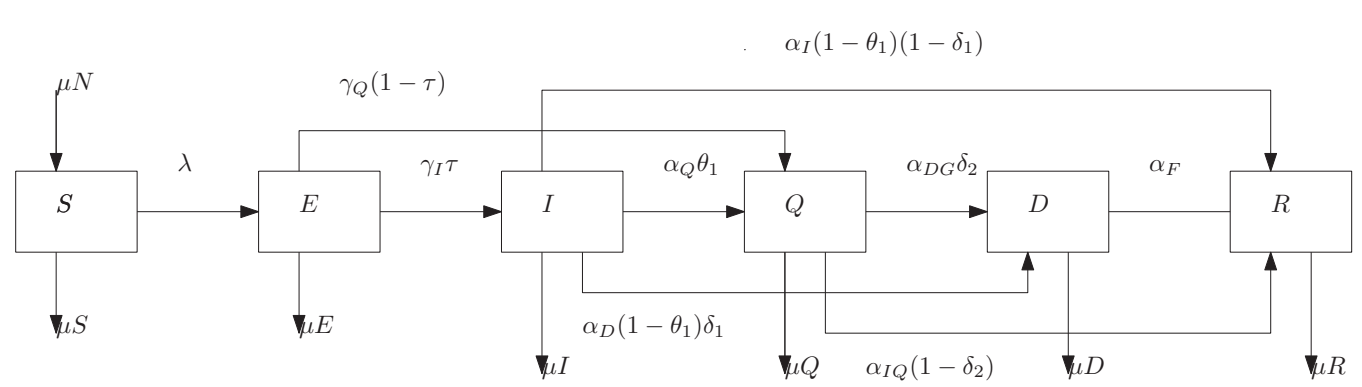

Figure 11. The SEIQDR compartmental model

$$
\begin{aligned}
S^{\prime} & =\mu N-\frac{\beta_{I} S I+\beta_{D} S D}{N}-\mu S, \\
E^{\prime} & =\frac{\beta_{I} S I+\beta_{D} S D}{N}-\left[\gamma_{I} \tau+\gamma_{Q}(1-\tau)+\mu\right] E, \\
I^{\prime} & =\gamma_{I} \tau E-\left[\alpha_{Q} \theta_{1}+\alpha_{D}\left(1-\theta_{1}\right) \delta_{1}+\alpha_{I}\left(1-\theta_{1}\right)\left(1-\delta_{1}\right)+\mu\right] I, \\
Q^{\prime} & =\alpha_{Q} \theta_{1} I+\gamma_{Q}(1-\tau) E-\left[\alpha_{D Q} \delta_{2}+\alpha_{I Q}\left(1-\delta_{2}\right)+\mu\right] Q, \\
D^{\prime} & =\alpha_{D}\left(1-\theta_{1}\right) \delta_{1} I+\alpha_{D Q} \delta_{2} Q-\left[\alpha_{D}+\mu\right] D, \\
R^{\prime} & =\alpha_{I}\left(1-\theta_{1}\right)\left(1-\delta_{1}\right) I+\alpha_{I Q}\left(1-\delta_{2}\right) Q+\alpha_{D} D-\mu R,
\end{aligned}
$$

with the non-negativity requirements of the initial conditions:

$$
S(0) \geq 0, \quad E(0) \geq 0, \quad I(0) \geq 0, \quad Q(0) \geq 0, \quad D(0) \geq 0, \quad R(0) \geq 0 .
$$

\subsection{Numerical Simulations}

We do a numerical simulation to illustrate the above theory. We begin with the SEIHDR model (no quarantine) with parameters

$$
\begin{aligned}
\mu & =0.008, \quad \beta_{I}=0.650, \quad \beta_{H}=0.540, \quad \beta_{D}=0.330, \quad \gamma=0.83, \\
\alpha_{H} & =0.309, \quad \alpha_{D}=0.075, \alpha_{I}=0.067, \quad \alpha_{D H}=0.099, \\
\alpha_{I H} & =0.063, \quad \theta_{1}=0.197, \quad \delta_{1}=0.50, \quad \delta_{2}=0.50, \quad N=1 .
\end{aligned}
$$

For these values the reproduction number $R_{0}$ for the SEIHDR model is

$$
R_{0}=10.02>1,
$$

so the endemic equilibrium is stable.

Using the same parameter values as (12) and the quarantine parameter values

$$
\begin{aligned}
\beta_{D} & =0.15, \gamma_{I}=0.76, \gamma_{Q}=0.50, \tau=0.50, \alpha_{Q}=0.309, \quad \alpha_{D Q}=0.099, \\
\alpha_{I Q} & =0.063, \theta_{1}=0.197, \delta_{1}=0.50, \delta_{2}=0.50, \quad N=1 .
\end{aligned}
$$

We get the reproduction number to be:

$$
R_{Q}=3.75
$$


It can be seen that there is a substantial decline in the level of infection upon applying quarantine.

Fig 12 shows the numerical simulation of the SEIHDR model (5) (without quarantine). Fig 13 shows the numerical simulation of the SEIQDR model (10) with the reduced level of infection.

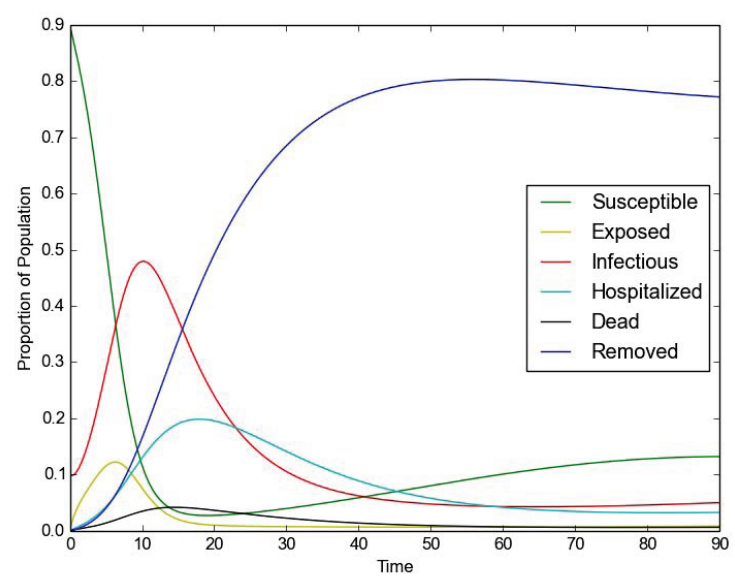

Figure 12. Numerical simulation of the SEIHDR model (5) (no quarantine).

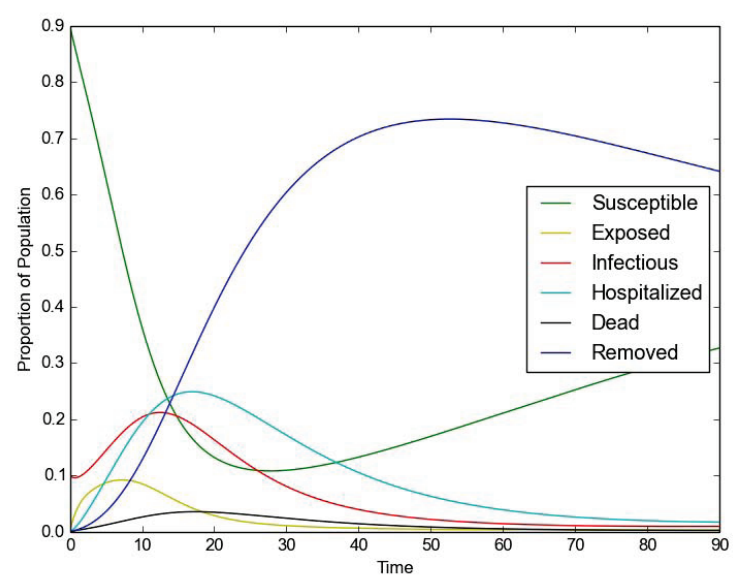

Figure 13. Numerical simulation of the SEIQDR model (10) showing the reduced level of infection.

\subsection{Comparing the SEIQDR Model to WHO Data}

In this section, we compare the SEIQDR model to the data of confirmed cases for the 2014 Ebola outbreak in Liberia (WHO, 2015b) provided by the World Health Organisation. Figure 14 compares the infected $\mathrm{I}(\mathrm{t})$ obtained from the SEIQDR model to the WHO data.

\section{The SEIQDR Model with Vaccination}

In this section, we study the SEIQDR model with vaccination similar to the analysis in Section 5.

\subsection{The Compartmental Model with Vaccination}

In this section, we modify the SEIQDR model by adding a vaccination term $v$. Figure 15 shows the relationship between the variables of the SEIQDR model with vaccination.

The system of equations that describe the SEIQDR model with vaccination are: 


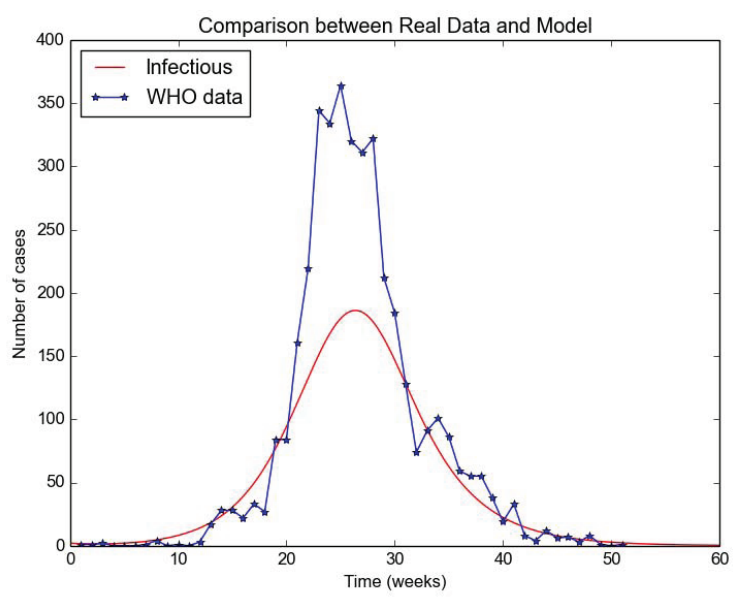

Figure 14. Graph of infected I(t) obtained from (10) with $\mu=0.0001, \beta_{I}=0.0002, \beta_{H}=0.0035, \beta_{D}=0.010, \gamma_{I}=0.83$, $\gamma_{Q}=0.2, \alpha_{Q}=0.540, \alpha_{D}=0.095, \alpha_{I}=0.002, \alpha_{D Q}=0.099, \alpha_{I Q}=0.063, \theta_{1}=0.385, \delta_{1}=0.0003, \delta_{2}=0.500, \tau=0.9$, $S(0)=600, E(0)=0, I(0)=2, H(0)=0, D(0)=0$, and $R(0)=0$ versus the real data of confirmed cases for the 2014 Ebola outbreak occurred in Liberia.

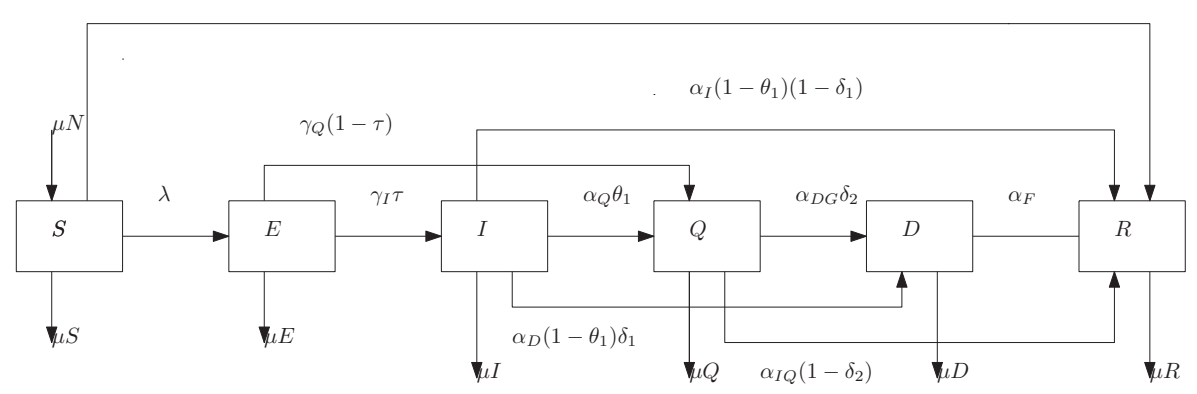

Figure 15. The SEIQDR compartmental model with vaccination.

$$
\begin{aligned}
S^{\prime} & =\mu N-\frac{\beta_{I} S I+\beta_{D} S D}{N}-\mu S-v S, \\
E^{\prime} & =\frac{\beta_{I} S I+\beta_{D} S D}{N}-\left[\gamma_{I} \tau+\gamma_{Q}(1-\tau)+\mu\right] E, \\
I^{\prime} & =\gamma_{I} \tau E-\left[\alpha_{Q} \theta_{1}+\alpha_{D}\left(1-\theta_{1}\right) \delta_{1}+\alpha_{I}\left(1-\theta_{1}\right)\left(1-\delta_{1}\right)+\mu\right] I, \\
Q^{\prime} & =\alpha_{Q} \theta_{1} I+\gamma_{Q}(1-\tau) E-\left[\alpha_{D Q} \delta_{2}+\alpha_{I Q}\left(1-\delta_{2}\right)+\mu\right] Q, \\
D^{\prime} & =\alpha_{D}\left(1-\theta_{1}\right) \delta_{1} I+\alpha_{D Q} \delta_{2} Q-\left[\alpha_{D}+\mu\right] D, \\
R^{\prime} & =\alpha_{I}\left(1-\theta_{1}\right)\left(1-\delta_{1}\right) I+\alpha_{I Q}\left(1-\delta_{2}\right) Q+\alpha_{D} D-\mu R+v S,
\end{aligned}
$$

where $v$ is the percentage of individuals vaccinated.

\subsection{Numerical Simulations in Case of Vaccination}

We simulate the SEIQDR model with vaccination (16) in order to visualise and predict the evolution of every class of individuals in case of vaccination (Rachah \& Torres, 2015). This study focusses on the test of different rates of vaccination and how they affect the curve of every class. We will consider the proportion of susceptible, exposed, infectious, quarantined, dead or removed (ie. $s=\frac{S}{N}, e=\frac{E}{N}, i=\frac{I}{N}, q=\frac{Q}{N}, d=\frac{D}{N}, r=\frac{R}{N}$ so that the total population is $N=1$ ).

The results are shown in Figures 17 and 18. These figures show different rates of vaccination and their effect on the peak of the curve of the infected individuals with time. The curve of the infected class shows that when the percentage of vaccinated individuals increases the peak of the curve of infected class goes down indicating that less individuals are infected and the period of infection is shortened while the number of removed individuals go up. 
In fact, when there is an outbreak without vaccination (only quarantine), as in the case of Section 6.4 the disease persist in the population for a longer period of time (see Figure 12), while in the presence of vaccination the disease suffers extinct in a short period of time (see Figure 18). This shows the efficiency of using vaccination strategy as a means of Ebola outbreak control.

Table 4 shows the percentage (to the nearest whole number) of infected individuals i corresponding to different rates of vaccination $v$ and the respective number of weeks for the peak of the $i(t)$ curve to be reached.

Table 4. Maximum percentage infected i corresponding to different rates of vaccination $v$ and the respective number of weeks for the peak of the $i(t)$ curve to be reached.

\begin{tabular}{|c|c|c|}
\hline Rate of vaccination & Maximum percentage infected & Weeks to the peak of $\mathrm{i}(\mathrm{t})$ \\
\hline 0 & $21 \%$ & 12.3 \\
0.025 & $18 \%$ & 10.6 \\
0.05 & $15.3 \%$ & 9.4 \\
0.10 & $13 \%$ & 7.5 \\
0.15 & $11.4 \%$ & 6.2 \\
0.20 & $10.7 \%$ & 5.1 \\
0.30 & $9.9 \%$ & 4.0 \\
0.40 & $9.1 \%$ & 3.5 \\
\hline
\end{tabular}

It can be seen from Table 4 that as the rate of vaccination increases the maximum percentage of individuals infected also increases. Increasing $v$ between 0.15 and 0.4 , the percentage infected increases by a small margin. Figure 16 shows the relation between the rate of vaccination $v$ and the maximum individuals infected $\mathrm{i}$ by the disease.

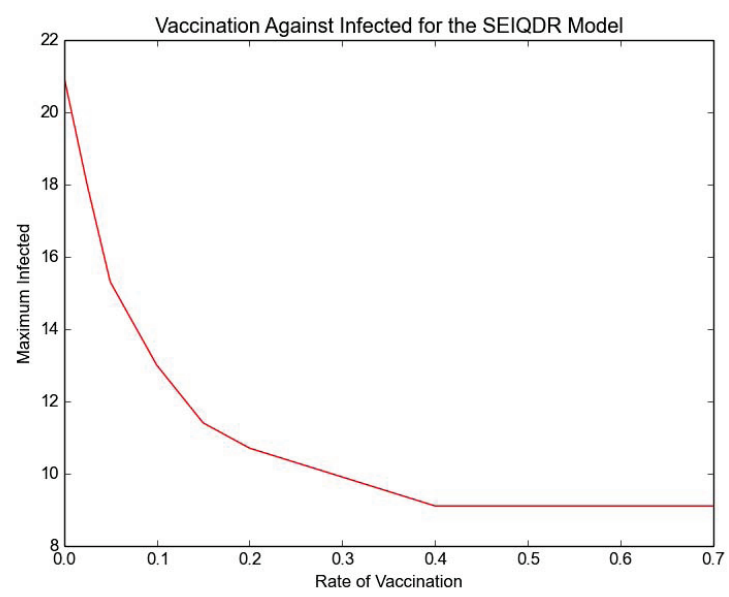

Figure 16. A graph showing the relation between the rate of vaccination and the maximum percentage of individuals infected for the SEIQDR model.

From Figure 16, the threshold proportion of population to be vaccinated to control the disease is estimated to be not less than $v=0.35$. 


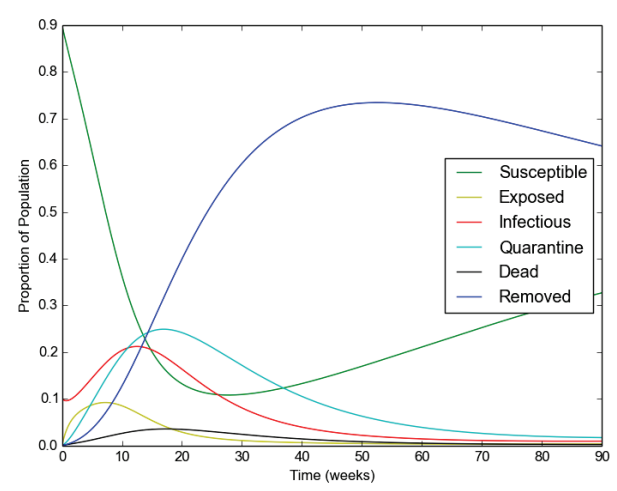

(a)

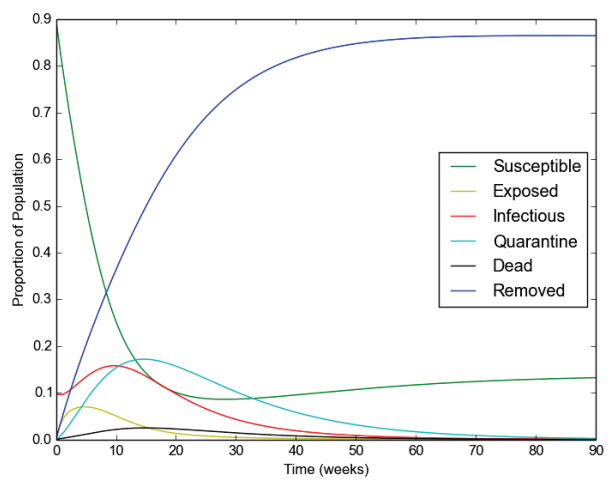

(c)

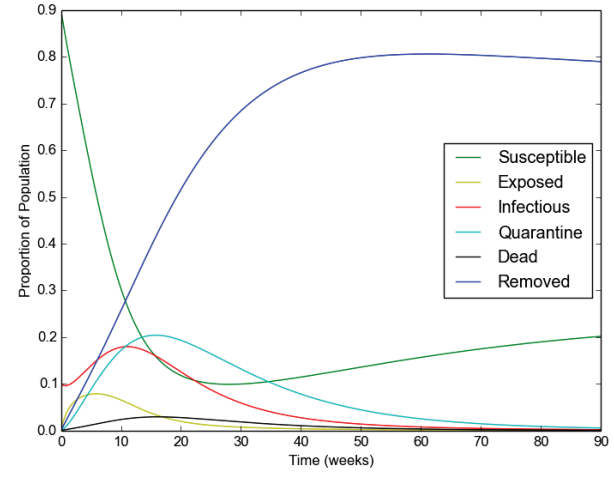

(b)

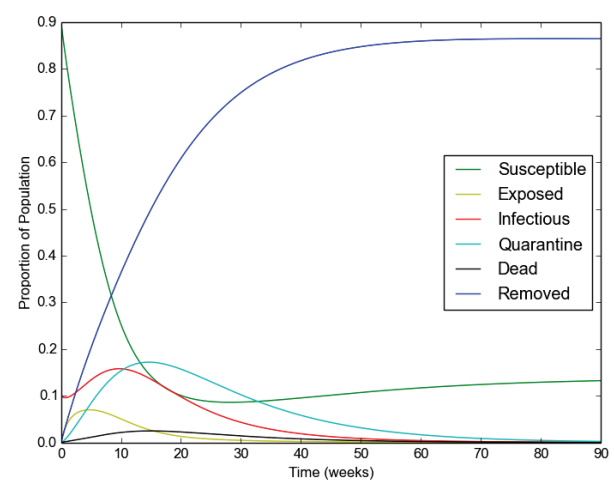

(d)

Figure 17. Numerical simulations of the SEIQDR model (16) with parameter values same as that of Figure 13 and different rates of vaccination: $v=0(\mathrm{a}), v=0.025(\mathrm{~b}), v=0.05(\mathrm{c}), v=0.10(\mathrm{~d})$.

\section{Conclusion}

In this paper, the SEIR model which does not consider the post-mortem contact effect and the SEIHDR model which takes into account the post mortem contact effect on the spread of the Ebola virus disease were analysed. For the first model, by constructing the equilibria and stability analysis, it was found that if the basic reproduction number $R_{0}<1$, then the disease-free equilibrium is stable. That is, the disease dies out. If $R_{0}>1$, then the endemic equilibrium is stable showing that the disease is present and persist in the population. Secondly, we analysed the SEIHDR model which is a modification of the SEIR model and which takes into account hospitalized and dead individuals regarding them as infectious. We compared both the SEIR and the SEIHDR models to the WHO data of confirmed cases for the 2014 Ebola outbreak in Liberia. It was observed that the results of the SEIHDR model agrees better with the WHO data than that of the SEIR model especially regarding the early stages of the outbreak. This is reasonable since it shows that Ebola patients were infected more through post-mortem contact during the first few weeks as a result of the initial misunderstanding and misdiagnoses of the Ebola virus. This further indicates that the post-mortem contact effect is important in the dynamics of the Ebola disease. We also formulated and analyzed the SEIQDR model which is a modification of the SEIHDR model which incorporates quarantine as an intervention regarded as inclusive of hospitalization and supervised burials. It was shown that the results of the SEIQDR model agrees better with the WHO data than that of the SEIR and the SEIHDR models especially during the latter stages of the disease outbreak. This is apparently due to the fact that knowledge about the spread of the disease increased with time and also began to effectively introduce interventions (mainly quarantine) to control the spread. It is therefore quite possible that if quarantine (in our broader sense) had been introduced earlier, the disease would not have affected so many people since the SEIQDR model indicate a far lower peak of infectives than the WHO data. It is therefore concluded that quarantine in its own right is an efficacious intervention. Finally, we modified both the SEIHDR and the SEIQDR models by introducing vaccination as an intervention. The modified SEIQDR indicates 


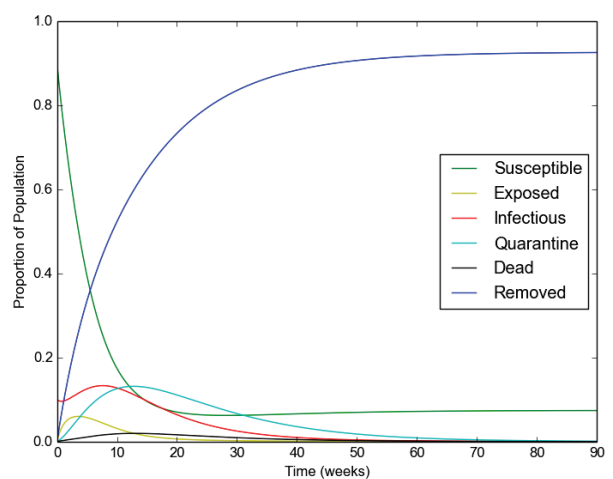

(a)

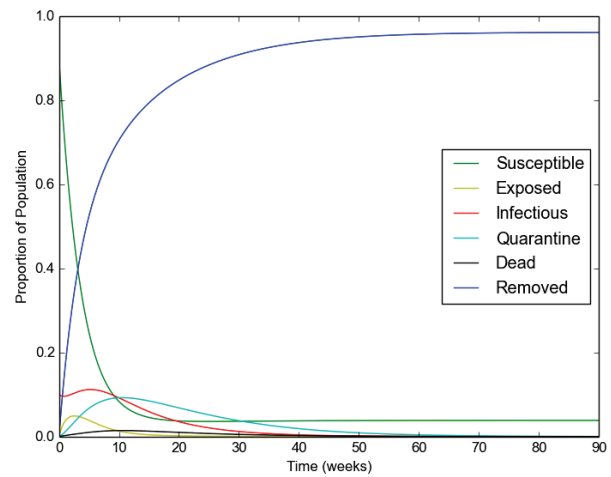

(c)

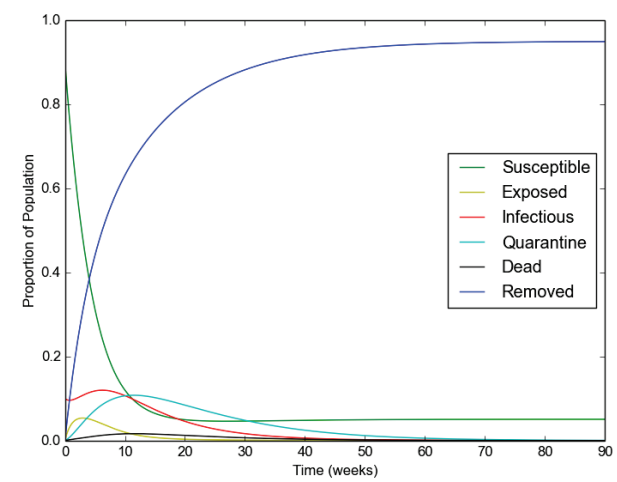

(b)

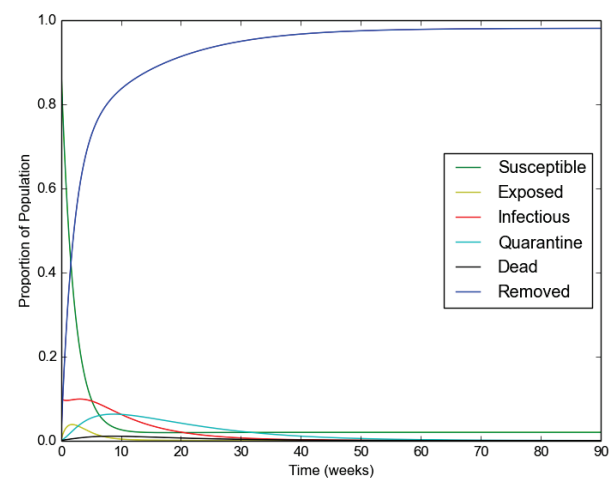

(d)

Figure 18. Numerical simulations of the SEIQDR model (16) with parameter values same as that of Figure 13 and different rates of vaccination: $v=0.15(\mathrm{a}), v=0.20(\mathrm{~b}), v=0.30$ (c), $v=0.4$ (d).

a lower threshold which should not be less than $25 \%$ as compared to that of the SEIHDR model which should not be less than $65 \%$. It was observed, moreover, that with vaccination, the disease suffers extinction in a much shorter period of time. Thus, it offers an additional strategy to control the disease more efficiently.

\section{References}

Astacio, J., Briere, D. M., Guilln, M., Martinez, J., Rodriguez, F., \& Valenzuela-Campos, N. (1996). Mathematical models to study the outbreaks of Ebola. Biometrics Unit Technical Reports BU-1365-M; Cornell University: New York, NY, USA, pp. 1-18.

Britton, N. (2012). Essential mathematical biology. Springer Science E Business Media.

CDC (2014). Recommendations for Breastfeeding/Infant Feeding in the Context of Ebola.

CDC (2015a). About Ebola Virus Disease. http://www.cdc.gov/vhf/ebola/about.html, Accessed January 2016.

CDC (2015b). Preliminary study finds that Ebola virus fragments can persist in the semen of some survivors for at least nine months. http://www.cdc.gov/media/releases/2015/p1014-ebola-virus.html, Accessed January 2016.

Chowell-Puente, G. (2005). Mathematical Models of Emergent and Re-emergent Infectious Diseases: Assessing the Effects of Public Health Interventions on Disease Spread, (Doctoral dissertation, Cornell University).

Chowell, D., Castillo-Chavez, C., Krishna, S., Qiu, X., \& Anderson, K. S. (2015). Modelling the effect of early detection of Ebola. The Lancet Infectious Diseases, 15(2), 148-149. http://dx.doi.org/10.1016/S1473-3099(14)71084-9

Funk, D. J., \& Kumar, A. (2015). Ebola virus disease: an update for anesthesiologists and intensivists. Canadian Journal of Anesthesia/Journal canadien d'anesthsie, 62(1), 80-91. 
Gatherer, D. (2014). The 2014 Ebola virus disease outbreak in West Africa. Journal of General Virology, 95(8), 16191624. http://dx.doi.org/10.1099/vir.0.067199-0

Keeling, M. J., \& Rohani, P. (2008). Modeling infectious diseases in humans and animals. Princeton University Press.

Khanh, N. H. (2015). Stability analysis of an influenza virus model with disease resistance. Journal of the Egyptian Mathematical Society.

Khan, M. A., Ali, Z., Dennis, L. C. C., Khan, I., Islam, S., Ullah, M., \& Gul, T. (2015). Stability Analysis of an SVIR Epidemic Model with Non-linear Saturated Incidence Rate. Applied Mathematical Sciences, 9(23), 1145-1158.

Legrand, J., Grais, R. F., Boelle, P. Y., Valleron, A. J., \& Flahault, A. (2007). Understanding the dynamics of Ebola epidemics, Epidemiology and infection, 135(04), 610-621. http://dx.doi.org/10.1017/S0950268806007217

Mamo, D. K., \& Koya, P. R. (2015). Mathematical Modeling and Simulation Study of SEIR disease and Data Fitting of Ebola Epidemic spreading in West Africa. Journal of Multidisciplinary Engineering Science and Technology (JMEST) ISSN, 3159-0040.

Oduro, F., Okyere, G. A., \& Azu-Tungmah, G. T. (2012). Transmission dynamics of Malaria in ghana. Journal of Mathematics Research, 4(6), 22. http://dx.doi.org/10.5539/jmr.v4n6p22

Oduro, F., \& Apaaboah, G. (2016). Optimal Control of Ebola Transmission Dynamics with Quarantine and Vaccination. Unpublished manuscript.

Okeke, I. N., Manning, R. S., \& Pfeiffer, T. (2014). Diagnostic schemes for reducing epidemic size of african viral hemorrhagic fever outbreaks. The Journal of Infection in Developing Countries, 8(09), 1148-1159. http://dx.doi.org/10.3855/jidc.4636

Pan American Health Organization, World Health Organization (2014). Ebola virus disease (EVD), implications of introduction in the Americas (Accessed January 2016) http://www.paho.org/hq/index.php?option=com_docman\&task=doc_view\&gid=26413+\&Itemid=999999\&lang=en

Rachah, A., \& Torres, D. F. (2015). Mathematical modelling, simulation, and optimal control of the 2014 Ebola outbreak in West Africa. Discrete Dynamics in Nature and Society.

Rivers, C. M., Lofgren, E. T., Marathe, M., Eubank, S., \& Lewis, B. L. (2014). Modeling the impact of interventions on an epidemic of Ebola in Sierra Leone and Liberia. PLoS currents, 6. http://dx.doi.org/10.1371/currents.outbreaks.4d41fe5d6c05e9df30ddce33c66d084c

Van den Driessche, P., \& Watmough, J. (2002). Reproduction numbers and sub-threshold endemic equilibria for compartmental models of disease transmission. Mathematical biosciences, 180(1), 29-48. http://dx.doi.org/10.1016/S00255564(02)00108-6

WHO (2015a). Ebola virus disease Fact sheet $N^{0} 103$ Updated August 2015. http://www.who.int/mediacentre/factsheets/fs103/en/, Accessed January 2016.

WHO (2015b). Ebola data and statistics. http://apps.who.int/gho/data/node.ebola-sitrep.quick-downloads?lang=en, Accessed January 2016.

WHO (2015c). Ebola situation report 9 December 2015, http://apps.who.int/ebola/current-situation/ebola-situation-report9-december-2015, Accessed January 2016.

WHO (2016). Ebola virus disease Fact sheet $N^{0} 103$ Updated January 2016. http://www.who.int/mediacentre/factsheets/fs103/en/, Accessed January 2016.

Wikipedia, the Free Encyclopedia. Ebola virus disease. https://en.wikipedia.org/wiki/Ebola_virus_disease\#cite_noteWHO2014-1, Accessed January 2016.

Yamin, D., Gertler, S., Ndeffo-Mbah, M. L., Skrip, L. A., Fallah, M., Nyenswah, T. G., Altice, F. L., \& Galvani, A. P. (2015). Effect of Ebola progression on transmission and control in Liberia. Annals of internal medicine, 162(1), $11-17$.

\section{Copyrights}

Copyright for this article is retained by the author(s), with first publication rights granted to the journal.

This is an open-access article distributed under the terms and conditions of the Creative Commons Attribution license (http://creativecommons.org/licenses/by/4.0/). 